\title{
LOCALIZAÇÃO ESPECIAL DE ESTÍMULOS SONOROS EM INDIVÍDUOS CEGOS CONGÊNITOS: ESTUDO COMPARATIVO DA POSIÇÃO TRIDIMENSIONAL DA CABEÇA EM ADULTOS CEGOS CONGÊNITOS E INDIVÍDUOS VIDENTES
}

SPATIAL LOCALIZATION OF SOUNDS IN BLIND INDIVIDUALS. COM PARATIVE STUDY OF THREE DIMENSIONAL POSITION OF THE HEAD IN BLIND AND NON BLIND ADULTS

\author{
Juliana Gonçalves da Silva GERENTE ${ }^{1}$ \\ Augusto Gil PASCOAL ${ }^{2}$ \\ Maria Leonor Moniz PEREIRA ${ }^{3}$
}

RESU M O: a capacidade para localizar objetos fixos ou em movimento no espaço tridimensional depende da função visual. No indivíduo cego, as modalidades sensoriais remanescentes, nomeadamentea audição, poderiam compensar a visão na local ização espacial. O objetivo deste estudo foi analisar o papel da audição no mecanismo de localização espacial por meio da habilidade de orientar de forma precisa a cabeça face à fonte sonora. Cinco adultos cegos congênitos foram comparados com cinco sujeitos videntes vendados. A tarefa consistiu na orientação da cabeça ao estímulo sonoro, emitido por sete fontes diferentes, com localização fixa. A posição tridimensional da cabeça e tronco foi registrada por um sistema de varredura eletromagnético (Flock of Birds System). Para cada som produzido foi calculado o "erro de localização". Esta medida correspondeu à diferença entre o registro da posição obtido durante o teste e durante uma posição de controle. Os resultados revelaram que nos indivíduos cegos congênitos a magnitude de erro de localização dos estímulos auditivos foi superior aos indivíduos videntes. Conclui-se que a representação mental formada com base na visão constitui um dos prérequisitos para um bom desempenho nas tarefas espaciais.

PALAVRAS-CHAVES: percepção espacial; cegueira; percepção auditiva, educação especial.

A BSTRACT: the ability to locate stationary or moving objects in space depends on visual function. It is thought that for blind individuals, the remaining sensory modalities, in particular hearing, will compensate for the absence of vision in spatial localization. This study aimed to analyzetherole of hearing on thespatial localization mechanism by looking at the ability to accurately direct the head to the source of sound. Five congenitally blind adults were compared to five sighted people who wore blindfolds. The task consisted of turning the head toward the sound stimulus, coming from seven different fixed point sources. The three dimensional position of the head and trunk was registered by an electromagnetic scanning device (Flock of Birds System). The "error of localization" was calculated for each sound produced. This measurement corresponded to the difference between the recorded positions during the test and the control position. The results revealed that in congenitally blinded individuals the magnitude of the "error of localization" was greater than in sighted individuals. The conclusion was that mental representations formed based on vision constitutes one of the prerequisites to good performance on spatial tasks.

KEYWORDS: sound perception; blindness; hearing; special education.

\footnotetext{
${ }^{1}$ Fisioterapeuta - Mestre em Reabilitação na Especialidade de Deficiência Visual - Universidade Técnica de Lisboa - Faculdade de Motricidade Humana - julianagerente@terra.com.br

${ }^{2}$ Fisioterapeuta - Doutor em Motricidade Humana na Especialidade de Ciências da Motricidade pela Universidade Técnica de Lisboa. Faculdade de Motricidade Humana, Universidade Técnica de Lisboa. Departamento de Ciências da Motricidade. gpascoal @fmh.utl.pt

${ }^{3}$ Doutora em Ciências da Motricidade H umana na área de Educação Especial e Reabilitação pela Universidade Técnica de Lisboa. Departamento de Educação Especial e Reabilitação. Universidade Técnica de Lisboa Faculdade de Motricidade Humana- Impereira@fmh.utl.pt
} 


\section{INTRODUÇÃO}

A habilidade espacial representa a capacidade de percepção das referências de posição e orientação dos objetos no espaço e a utilização deste conhecimento na resolução de problemas do cotidiano (MORATO, 1989). A capacidade de representação do espaço é adquirida em informações oriundas de diversas modalidades sensoriais, as quais nos permitem a compreensão e referenciação no espaço tridimensional.

Na ausência de visão, como aconteceem sujeitos portadores de cegueira, a representação espacial subjacente ao processo de localização e referenciação espaciais, éo resultado da convergência das aferências auditivas, proprioceptivas, vestibulares e táteis. O modo de processamento apresentaria características específicas, combinando informações proporcionadas para cada uma das modalidades sensoriais remanescentes, de forma a ser possível a construção de uma representação mental do espaço. Com efeito, o cego utiliza preferencialmente a audição quando é necessário recol her informações do espaço, nomeadamente a localização de objetos. Assim, em face a uma fonte sonora fixa, o cego realiza movimentos da cabeça em todas as direções no sentido de conseguir al inhamentos de ambas as orel has face ao som e deste modo criar diferenças binaurais que lhe permitam estabelecer uma primeira identificação do som. As informações proprioceptivas e vestibulares associadas ao movimento da cabeça permitem referenciar este segmento no conjunto dos restantes segmentos corporais, nomeadamente o tronco. Este processo de referenciação acontece de acordo com uma representação do corpo (esquema corporal) e que é utilizado de forma particularmente eficienteno cego nos seus deslocamentos num espaço previamente mapeado por estímulos sonoros.

O refinamento dos sentidos remanescentes, nomeadamentea audição, pareceexplicar o desenvolvimento de competências em audição espacial por parte do cego tal, como tem sido sugerido por alguns estudos (ASHMEAD; DAVIS; NORTHIN GTON, 1995; LESSARD; PARE, 1998; LEWALD, 2002). Contudo, esta possibilidade tem sido contestada com o argumento que a cegueira precoce pode impedir o completo desenvolvimento das restantes modalidades sensoriais.

A literatura não apresenta sistematização dequemodo estemecanismo é utilizado nos cegos para a representação espacial. A comparação entre sujeitos cegos e videntes tem revel ado que os cegos apresentam poucas diferenças quanto à local ização de estímulos auditivos (LEWA LD, 2002), apesar de se ter verificado que as crianças cegas podem discriminar melhor as mudanças na elevação e distância do som (ASH MEAD; DAVIS; NORTHINGTON, 1995). 


\section{MÉTOdo}

A amostra foi constituída por dois grupos: grupo de cegos congênitos, que integrou cinco adultos cegos por glaucoma congênito, duas mulheres e três homens; idade $=44.8 \pm 7.26$ anos); e grupo de videntes, igualmente de ambos os sexos ( 2 mulheres e 3 homens; idade $=46 \pm 13$.1 anos). Para o conceito de cegueira adotamos as resoluções do Consel ho Internacional de Oftal mologia daW orld V ision Community (2002). Todos os participantes da amostra estavam dinicamenteestáveis, sem queixas auditivas tendo dado consentimento informado em participar no estudo.

O sujeito em teste foi posicionado no centro de uma circunferência de $320 \mathrm{~cm}$ de diâmetro em cuja periferia fora colocados alto-falantes a uma altura de $130 \mathrm{~cm}$ com a seguinte disposição: três alto-fal antes local izados à frente do sujeito e separados $30 \mathrm{~cm}$ entre si; e dois alto-falantes localizados respectivamente na lateral direita e esquerda do sujeito. Os alto-falantes foram ativados seqüencialmentede modo a produzir um som contínuo puro deintensidadesonora de 75 decibéis efreqüência de $512 \mathrm{~Hz}$, com duração decinco segundos. A seqüência de som correspondeu à seguinte localização dos alto-fal antes: AFF (alto-falante frontal frontal), ALD (alto-falante lateral direito), ALE (alto-falante lateral esquerdo), ALD (alto-falantelateral direito); AFD (alto-falantefrontal direito), AFF (al to-falante frontal frontal), AFE (al to falante frontal esquerdo). O alto-falanteAFF foi al inhado com a cabeça (nariz) do sujeito.

Com os sujeitos na posição sentada (mãos apoiadas na coxa), foi pedido que orientassem a cabeça ("oriente o seu nariz") com o som produzido em cada altofalante e que no período de produção do som fixasse a cabeça nessa direção. Os sujeitos videntes realizaram duas repetições do teste com a visão restringida por meio da utilização de uma venda sobre os ol hos e duas repetições sem limitação da visão (sem venda). N as repetições sem venda, foi pedido queo sujeito marcasse o momento em que a cabeça se encontrava perfeitamente alinhada com a fonte sonora.

A posição tridimensional da cabeça e tronco foi registrada em cada local ização sonora durante \pm 5.5 segundos com auxílio de um sistema de varredura eletromagnético (Flock of Birds System, A scension T echnol ogy Inc., Burlington, U SA, www.ascension-tech.com) otimizado por um software específico (M otion M onitor v.6.05 www.innsport.com). Este sistema é composto por sensores eletromagnéticos cuja local ização e orientação pode ser determinada quando estes se encontram no interior de um campo eletromagnético calibrado gerado por um transmissor de longo alcance. Foram utilizados dois sensores el etromagnéticos: o sensor do tórax, colocado no processo espinhoso da primeira vértebra torácica; e o sensor da cabeça colocado sobre o occipital. Um terceiro sensor eletromagnético foi utilizado no registro da posição tridimensional de referências ósseas do tórax e cabeça, previamente identificadas por pal pação e posteriormente utilizadas na definição de sistemas de coordenadas locais no tórax e na cabeça. 
A posição 3D do tórax e da cabeça foram calculadas a partir da orientação dos respectivos sistemas de coordenadas locais relativamente ao transmissor local izado atrás do sujeito (sistema decoordenadas global). As rotações do tórax foram descritas sob a forma de em ângulos de Euler/ Cardan de acordo com o protocolo de registro cinemático proposto por Wu et al. (2005). Em cada local ização sonora foi cal culado o "erro delocal ização", correspondenteà diferença entre as rotações da cabeça e do tórax no registro de referência (posição de normal ização) ea média dos valores de rotação registrados entre o quarto equinto segundo em cada som da seqüência sonora. N uma das repetições sem venda, foi pedido aos sujeitos que marcassem o momento em que a cabeça estava no mel hor alinhamento da fonte sonora para cada um dos sons produzidos na seqüência sonora. A marcação foi realizada por intermédio de um sinal de referência introduzida num canal separado do registro cinemático. Esta repetição constituiu o registro de referência. Os sujeitos cegos realizaram três repetições do teste. $O$ registro de referência foi realizado com recurso a um observador que orientou manualmente a cabeça do sujeito com a fonte sonora, ao mesmo tempo em que procedia à marcação do sinal.

A variável "erro de localização" foi interpretada nas componentes de "magnitude do erro de localização" e "direção do erro de localização". Todos os dados foram exportados para formato compatível ao processamento eanáliseatravés de rotinas de programação em M atlab (Mathwoks, USA, www.Mathwoks.com).

Em cada localização dos al to-falantes, as posições 3D da cabeça e do tórax foram comparadas entre os grupos com recurso ao teste T de Student para amostras independentes eM ann W hitney U . Para cada grupo, a magnitude do erro delocalização observado no conjunto dos sons de orientação frontal, para em cada rotação da cabeça e tórax, foram comparados com a magnitude do erro encontrado nos sons de orientação lateral. Esta comparação foi realizada com recurso ao teste $T$ deStudent para amostras emparelhadas. Todo o processamento foi realizado com auxílio de software específico (SPSS), para um nível de significância de 0,05.

\section{RESULTADOS}

Nesteestudo, foi considerada a variável dependente "erro delocalização" subdividida em duas variáveis complementares: magnitude do erro, referenteaos valores em absoluto do erro de localização (diferença entre o valor de rotação angular na posição denormalização eo valor registrado em cada local ização sonora) edireção do erro, ao sentido do desvio (erro) de orientação da cabeça relativamente à fonte sonora.

Os valores mais elevados de erro de localização encontram-se associados à rotação axial da cabeça e à rotação do tórax. Este comportamento deriva do fato desta categoria demovimento ser aquela que no presentedispositivo experimental apresenta maior amplitude de variação e, por isso, se encontrava 
mais exposta ao erro. $\mathrm{Na}$ localização sonora frontal-frontal, foram encontradas diferenças significativas entre os grupos na magnitude do erro de localização na rotação axial da cabeça $(p=.029)$, na incl inação da cabeça $(p=004)$ e na rotação do tórax $(p=.009)$. Na localização sonora lateral esquerda, os valores da magnitude do erro de local ização na rotação do tórax apresentaram diferenças estatisticamente significativa $(p=.028)$. Para a local ização sonora frontal direito, a magnitude do erro de local ização na rotação do tórax apresentaram diferenças estatisticamente significativas ( $p=.014)$. Na localização sonora frontal-frontal, a magnitude do erro de localização na rotação do tórax apresentou diferença estatisticamente significativa ( $p=.028)$. Quando a cabeça assume val ores negativos na rotação axial, inclinação, isso significa que os sujeitos assumiram uma posição mais em rotação esquerda, em inclinação esquerda da cabeça, relativamenteà posição dereferência. $\mathrm{Na}$ localização sonora frontal-frontal, foram verificadas diferenças significativas entreos grupos nos val ores de magnitudeerro delocalização de rotação e inclinação da cabeça, assim como na rotação do tórax. Os cegos tendem a posi cionar a cabeça mais em rotação axial direita eem inclinação lateral direita enquanto queos sujeitos videntes assumem uma posição mais em rotação axial esquerda e inclinação esquerda. Nesta localização sonora é, ainda, possível verificar que, em ambos os grupos, o erro delocal ização associado à rotação do tórax éno sentido de posicionar o tronco mais em rotação direita, comparativamente a posição de referência.

$\mathrm{N}$ a local ização sonora lateral esquerda, as diferenças na magnitude de erro de localização na rotação do tórax nos grupos apresentaram significado estatístico. Os cegos tendem a posicionar o tórax mais em rotação direita, enquanto que os videntes assumem uma posi ção do tronco mais em rotação esquerda. Para a localização sonora frontal direita, as diferenças no erro de localização na rotação do tórax nos grupos apresentaram significado estatístico. N esta localização sonora é possível verificar que, em ambos os grupos, o erro de localização associado à rotação do tórax é no sentido de posicionar o tronco mais em rotação direita, comparativamente a posição de referência.

\section{DıscusSÃo}

Os movimentos de rotação axial da cabeça e tórax foram os que apresentaram a maior variação da amplitude articular. Á partida, este resultado era esperado dada à local ização no mesmo plano dos estímul os sonoros. Contudo, seria razoável antecipar, que a estratégia para a localização dos sons com base na instrução dada aos sujeitos, "alinhamento do nariz com os alto-falantes", passasse, sobretudo pelo movimento da cabeça, sobre um tórax fixo. Mas tal não se veio a verificar eo envolvimento do tórax, por meio demovimentos derotação, constituiu uma estratégia seguida pela maioria dos sujeitos, com especial ênfasepara os cegos. Neste grupo, os valores de amplitude de rotação do tórax, assim como os de magnitude de erro de localização sonora, apresentaram-se mais el evados do que nos videntes. Sendo assim, estefato indica uma tendência que uma das estratégias 
adotadas pelos cegos no sentido de realizar a varredura de localização espacial foi a ampla movimentação do tórax, com manutenção da cabeça estável. Na orientação independente do individuo no espaço tridimensional é fundamental o domínio dos conceitos das partes do corpo e destas com o meio, e a correta utilização das estruturas corporais. Esta capacidade está na base do controle postural e no alinhamento dos vários segmentos corporais. No cego congênito, a regulação e controle da postura regem-se por outros mecanismos de referencial em relação a si e ao mundo que, segundo Fonseca (1999), tende a ser justificado pela privação sensorial do deficiente visual, e que o leva a valorizar as informações dos outros sentidos.

Em relação à magnitude de erro delocalização dos estímulos auditivos, Moura e Castro (1998) refere que os deficientes visuais apresentam dificuldades na atualização espacial. Com efeito, os videntes possuem uma imagem mental de referência dominada por imagens visuais queé decisiva para um bom desempenho em tarefas de localização espacial. Os cegos congênitos apresentam maiores dificuldades na representação mental do espaço, dada à ausência de uma estruturada representação do seu corpo e da relação que esteestabel ececom mundo exterior. Talvez seja essa a explicação para que os cegos apresentem valores de erro de localização da cabeça e tórax superiores aos registrados para os videntes. No grupo de cegos, o movimento do tórax poderia ser explicado pel o movimento em bloco e em conjunto com a cabeça. A deficiente utilização do esquema corporal devido a uma imagem corporal distorcida estaria na origem dos movimentos da cabeça e tronco em bloco, não havendo dissociação entre a cintura pélvica e a escapular. Em todas as localizações sonoras em teste, a magnitude de erro de local ização em rotação axial da cabeça apresentou valores superiores no grupo de cegos. Jeka; Bentzen; Lackner (1996) apresentam uma perspectiva idêntica ao verificarem que os cegos congênitos, freqüentemente, realizam tarefas espaciais com menor precisão do que os videntes, mesmo quando utilizam as informações auditivas.

$\mathrm{Na}$ orientação AFD, o grupo de cegos foi também aquele em que se verificou o maior envolvimento simultâneo do tórax através do movimento de rotação, reforçando o caráter indissociado do movimento da cabeça e do tórax. Mais uma vez, observa-se um padrão de movimento em bloco no grupo dos cegos congênitos, com possíveis conseqüências em alterações posturais futuras. Esta expectativa baseia-se nas observações de Cantanzariti e Salomez (2001) ao verificarem que a ocorrência de deformidades no tronco são maiores em deficientes visuais quando comparada com videntes. Se o erro de localização na rotação do tórax é menor que o da rotação axial da cabeça, e se o movimento da cabeça é em bloco com o tórax, isso podesignificar que os cegos tendem a orientar corretamente o tórax face à localização sonora. Assim, em lugar de alinharem o nariz com a fonte sonora, tendem a al inhar o tórax com o som assumindo uma rel ação estável eentre a cabeça etórax. A verificar-se esta possibilidade, há indicativos da presença de alterações posturais e perturbações do esquema corporal. Fjellvang e Solow 
(1986) relatam inúmeros fatores que podem induzir alterações posturais em indivíduos cegos: a falta de controleóptico, imagem corporal distorcida eincorreta percepção da vertical.

Foi observado que os cegos tendem a alinhar uma das orelhas, supostamentea orel ha dominante com a fonte sonora. N estas condições, o erro de localização na rotação axial da cabeça registrado estaria associado à discrepância na orientação do nariz eda orel ha predominante faceà localização da fonte sonora. Esta diferença estaria presente para as local izações sonoras de orientação frontal e lateral, dado que a discrepância se deve a erros de esquema corporal no que se refere à localização do nariz e da orel ha predominante. Esta interpretação parece ser corroborada pela ausência de diferenças significativas verificada no grupo de cegos e videntes, no erro de local ização de sons frontais quando comparado com sons laterais. Contudo, na orientação frontal (AFF[1]) os cegos apresentam diferenças estatisticamentesignificativas quando comparados aos videntes, no que se refere à rotação axial da cabeça. Este resultado parece confirmar que os cegos tendem a confiar na orelha predominante, alinhando-o com a fonte sonora, não conseguindo, no entanto fazer a correspondente compensação no sentido de alinharem o nariz, tal como as condições experimentais o exigiam. Tendo em consideração os resultados referentes à direção do erro, podemos afirmar que os cegos tendem a colocar a cabeça em rotação axial direita face à fonte sonora, mostrando quetalvez a orel ha esquerda seja a orel ha predominantepara a mai oria dos sujeitos. Blauert (1999) descreve que a forma cônica do pavilhão auricular funciona demodo a concentrar na pequena área do meato acústico a energia sonora que chega à sua superfície. O pavilhão auricular humano não é dotado de movimentos que Ihe permitam direcioná-lo em relação às fontes sonoras, então, esta função fica na dependência dos movimentos da cabeça.

Para as diferenças de erro de rotação axial da cabeça encontradas nas orientações AFF[1] eAFF[2], épossível atribuir ao sentido de desl ocamento da cabeça na seqüência de sons. Com efeito, a orientação $A F F[1]$ foi a primeira da seqüência enquanto que a AFF[2] foi a sexta da seqüência depois das orientações laterais e da orientação frontal direita. Este fato poderia explicar uma estruturação do esquema corporal e da calibração auditiva tendo, sobretudo, como referência a orientação AFD. Esta interpretação foi assinalada por Easton e Greene (1998) ao afirmarem que a informação auditiva pode ser importante para os cegos congênitos, que tendem a utilizála para a orientação espacial. Desta forma, épossível sugerir queem indivíduos cegos o feedback auditivo e motor substituem o sentido visual para cal ibrar o espaço auditivo. Desta maneira, quando ouvem o som AFF[2], os indivíduos cegos já executaram um reconhecimento espacial utilizando as pistas auditivas, tornando a rotação axial ea rotação do tronco mais similares aos videntes. Assim, al guns autores chegam a propor que o sistema de localização auditiva combina os in puts acústicos com a informação da posição da cabeça de forma a converter al vos acústicos numa imagem de referência espacial (TOLLIN; POPULIN, 2005). 
Os indivíduos cegos tendem a orientar o tórax faceà local ização sonora, assumindo um alinhamento da cabeça face ao tronco. Esta informação volta a reforçar a idéia quenos cegos há uma incorreta percepção da vertical, uma imagem corporal distorcida e um desalinhamento da postura. Idêntica opinião advoga Fernandes (1994) ao descrever que em geral o cego adota posturas inadequadas a uma concepção inadequada da vertical idade e por um conhecimento limitado do seu corpo e da percepção espacial. Verifica-se que o envolvimento do tórax é particularmente importante para as local izações sonoras frontais.

O grau de interdependência funcional determina que sempre que a cabeça gira em rotação axial é acompanhada em simultâneo pela inclinação da cervical baixa, para o mesmo lado, ea ind inação da cervical alta, para o lado oposto. Esperava-se que nas orientações sonoras laterais, o movimento de rotação axial da cabeça fosse acompanhado de incl inação lateral. Em ambos os grupos, a inclinação da cabeça apresenta valores entre -20 (inclinação à esquerda) e $20^{\circ}$ (inclinação à direita). Importa, no entanto, sal ientar que esta amplitude érelativa ao tórax, pelo que é necessário consi derar, também, os val ores de inclinação do tórax. É possível verificar que na localização da fonte sonora, o envolvimento do tórax é menor quando considerado com a contribuição da cabeça.

No que se refere à magnitude do erro de localização em inclinação da cabeça foi possível verificar diferenças significativas entre os dois grupos para a orientação frontal (AFF[1]). Os cegos apresentam dificuldades na percepção espacial eorientação, a qual pode ser atribuída mais a uma falta de experiências adequadas do que à inexistência da visão (JEKA; BENTZEN; LACKNER, 1996).

Desalientar quea magnitude de erro delocal ização somente apresenta diferenças na comparação entre cegos e videntes, na orientação AFF[1]. Na orientação sonora equival ente, AFF[2], estas diferenças não se verificaram. Este fato podeser integrado no mesmo conjunto de questões levantadas a propósito da rotação axial da cabeça, cujas respostas parecem estar associadas à desajustada estruturação do esquema corporal do cego congênito, nomeadamente no que se refere à localização espacial relativa dos segmentos cabeça e tórax.

\section{CONCLUSÃO}

A magnitude de erro de localização dos estímulos auditivos nos indivíduos cegos congênitos é superior à observada nos videntes. Numa análise conjunta das variáveis, foi constatado que nos cegos congênitos, o movimento do tórax aconteceu em bloco com a cabeça. Este fato indica uma tendência de má utilização do esquema corporal e de uma imagem corporal distorcida. A imagem do corpo, bem como sua relação com o meio ambiente, são conceitos abstratos para os deficientes visuais, porque eles não dispõem de referências visuais.

O estudo possibilitou evidenciar que nos movimentos de rotação axial da cabeça e rotação do tórax houve maior variação de amplitude articular. Esta movimentação 
indica que há uma varredura espacial, no sentido de cal ibrar o indivíduo no meio. Foi observado também, por meio de dados da pesquisa, a tendência que o cego congênito apresenta de posicionar a cabeça em função da orientação das orelhas face aos estímulos sonoros, e que tendem a alinhar a orel ha predominante com a fonte sonora. Ao analisar o resultado da variável direção do erro de localização, os resultados mostram que, os cegos tendem a posicionar mais a cabeça em rotação axial direita e inclinação lateral direita, e os videntes assumem uma posição mais em rotação axial à esquerda e inclinação à esquerda. Ainda, é possível verificar que os indivíduos cegos tendem a posicionar o tronco mais em rotação direita, comparativamentecom a posição de referência, enquanto que os sujeitos videntes assumem uma posição do tronco mais em rotação esquerda.

As alterações na posição da cabeça podem estar na origem dedisfunções cervicais com irradiação para o membro superior. Sendo assim, é de suma importância que o indivíduo cego seja orientado a não assumir erros posturais e ou ergonômicos, em virtude da privação do sentido visual.

\section{Referências}

ASHMEAD, D.; DA VIS, D.; N ORTHIN GTON , A. Contribution of listeners approaching motion to auditory distance perception. Journal of experimental psychology: $\mathrm{H}$ uman Perception \& performance, v.2, p.239-256, 1995.

BLAUERT, J. Spatial hearing: the psychophysics of human sound localization. London: The MIT Press, 1999.

CATANZA RITI, J.; SA LOMEZ, E. Visual Deficiency \& Scoliosis. Spine, n.1, p.48-52, 2001.

EASTON, R.D.; GREENE, A. A uditory cues for orientation \& postural control in sighted \& congenitally blind people. Exp. Brain Res, n.118, p.541-550, 1998.

FERN ANDES, J. Comportamento postural e deficiência visual: influência da prática desportiva dealto rendimento no comportamento postural deindivíduos com capacidadevisual nula e muito reduzida. 1994. Dissertação (Mestrado em Reabilitação na Especialidade de Deficiência Visual) - Faculdade de Motricidade Humana, Universi dade Técnica de Lisboa, Lisboa, 1994.

FJELLVANG, H.; SOLOW, B. Craniocervical postural relations \& craniofacial morphology in 30 blind subjects. A m J O rthod D entofacial O rthop. n. 4, p.327-346,1986.

FONSECA , V. P erturbações do D esenvol vimento eda A prendizagem - Tendências Filogenéticas eOntogenéticas. Cruz Quebrada: Faculdade de MotricidadeH umana, 1999.

JEKA , J.E.; BENTZEN , B.; LA CKNER, J. H aptic cues for orientation \& postural control in sighted \& blind individuals. Perception \& P sychophysics, n. 3, p.409-423, 1996.

LESSARD, N.; PARE, M. Early-blind human subjects localize sound sources better than sighted subjects. N ature, n.395, p.278-280, 1998. 
LEWALD, J. Opposing effects of head position on sound localization in blind \& sighted human subjects. Eur J N eurosci, v.15, p.1219-1224, 2002.

MORATO, P.P. D eficiência mental e aprendizagem. Estudo dos efeitos dediferentes ambientes de aprendizagem na aquisição de conceitos espaciais em crianças com trissomia 21. Lisboa: Universidade Técnica de Lisboa, 1989.

MOURA E CASTRO, J.A. Estudo da influência da capaci dade de resistência aeróbia na orientação e mobilidade do cego. Lisboa: Secretariado N acional para a Reabilitação e Integração das pessoas com deficiência, 1998.

TOLLIN, D.J.; POPULIN, C. Sound-localization performance in the cat: the effect of restraining the head. J N europhysiol, n. 93, p.1223-1234, 2005.

WU, G.F et al. ISB Recommendation on definitions of joint coordinate systems of various joints for the reporting of human joint motion. J. Biomech, n.38, p. 981-92, 2005. 